\title{
Pseudo-duplication of the optic nerve head
}

\section{LA Moore*}

\author{
Department of Optometry, Dublin Institute of Technology, Kevin Street, Dublin 8, Republic of Ireland
}

\author{
<Linda.Moore@dit.ie>
}

A 25-year-old woman presented for a routine eye examination. She felt that her distance and near vision was good, but experienced severe frontal headaches when reading or using a VDU (50 cm working distance) for prolonged periods. She had been wearing a prescription for reading and VDU work for four years, but had lost her spectacles. She reported that her old prescription was (RE) $0.75 \mathrm{D}$ and (LE) 0.50 $\mathrm{D}$ and that the wearing of this prescription reduced near asthenopic symptoms. She was using no medication and was in good general health at the time of the appointment and reported no personal or family history of ocular or systemic disease. Prior to commencement of ophthalmoscopy, she mentioned that an optometrist had told her previously that she had "some extra pigment at the back of the eye that should be looked at" by an ophthalmologist, but that she had never felt the need to seek further ophthalmological opinion.

Her unaided distance visual acuities were 6/5 monocularly and binocularly. Her distance subjective refraction was (RE) $-0.25-0.25$ x 60 (6/5) and (LE) pl $-0.25 \times 125$ (6/5). She had reduced amplitude of accommodation on push-up, measuring 4.50 $\mathrm{D}$ monocularly and binocularly and an near point of convergence (NPC) of $15 \mathrm{~cm}$. Fixation disparity testing using the Mallett unit at distance showed an orthophoric response, while testing at near showed a requirement for a 1 pd base-out prism. Monocular estimation method (MEM) retinoscopy showed a requirement of $0.75 \mathrm{D}$ of plus at near, resulting in a near prescription of (RE) $+0.50 /-0.25$ x 60 (N5) and (LE) $+0.75 /-0.25 \times 125$ (N5). Slitlamp biomicro- scopic examination of the anterior eye revealed mild blepharitis, with no apparent abnormalities of anterior ocular structures. Ophthalmoscopic findings of the optic disc and surrounding areas were confirmed by fundus photography. The right eye showed a pale excavation (Figure 1) approximately 1 disc diameter (DD) in size, situated 0.5 DD infero-nasally in relation to the optic disc, with the excavation linked to the optic disc by means of two retinal blood vessels. These blood vessels apparently pass over the excavation without entering it. The area immediately surrounding the lesion and between this and the optic disc is hypopigmented, with pigmentation at the margins of the lesion. No fundal anomalies were noted for the left eye. Other fundus structures were comparable for both eyes. A single field analysis SITA-FAST Central 24-2 threshold test using the Humphreys visual field analyser showed no field abnormalities present in either eye, with the fields for the two eyes comparable.

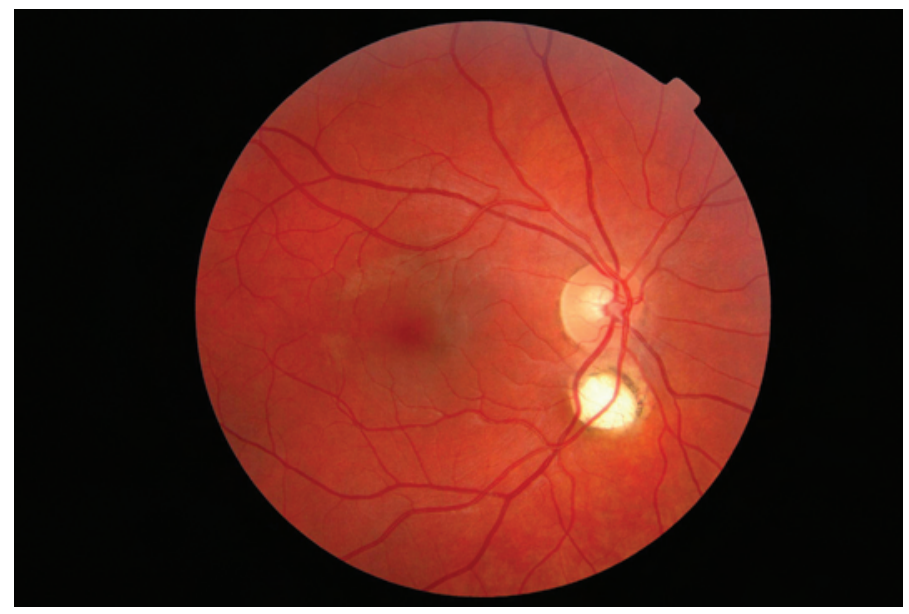

Figure 1. Fundus image of the RE 


\section{Discussion}

Pseudo-doubling of the optic nerve head is a rare condition, where a lesion resembling an optic disc is situated adjacent to the true optic disc ${ }^{1,2}$. Typical colobomas are located inferiorly and slightly nasally, resulting from failure of closure of the fetal fissure. This is the location of the coloboma in the case of the patient described above. The fetal fissure closes first in the region of the equator, with progressive closure anteriorly and posteriorly from that point. Failure of the fetal fissure to close posteriorly results in absence of choroid, pigment epithelium and retina in that area $^{3}$. This pseudo-duplication of optic discs may be caused by lesions such as optic disc coloboma, peripapillary chorioretinal coloboma or inflammatory foci ${ }^{3,4}$. According to Pesudovs and Weisinger (2000), the circumscribed disc-like appearance of this lesion, in combination with the presence of blood vessels and proximity to the true optic nerve head, differentiates it from other retinal lesions ${ }^{1}$. According to Taylor and Stout (1997), in pseudo-doubling of the optic disc, retinal vessels pass over the colobomatous area and large choroidal vessels are visible in the base of the coloboma $^{5}$, as is the case with this patient. These authors also discuss other ocular findings that may indicate the presence of other congenital abnormalities, but none of these extra findings were noted in this patient's case.

The findings in this case concur with those of Kamath et al. (1999), who published a case ${ }^{4}$ of peripapillary coloboma simulating a double optic disc, in that normal visual acuities and anterior segments were found. It is not thought to be an optic disc coloboma in that this structure is separate from the optic disc itself. Chorioretinal atrophy around the edge was viewed in the case of this patient, concurring with the findings ${ }^{6}$ of Barboni et al. (1998). Pigmentation of the coloboma in this case is consistent with findings of Islam et al. (2005) who reported this occurring in six out of eleven patients with this condition ${ }^{3}$. Comparison of this patient's fundus image with those published by Islam et al. (2005) show that it is likely that this lesion is caused by a peripapillary chorioretinal coloboma. More extensive field losses have been reported by Barboni et al. (1998) and Islam et al. (2005). However, Brink and Larson (1977) reported that in cases of true doubling of the optic disc, a double blind spot would be present ${ }^{7}$. The visual fields for this patient were comparable and did not show any field losses.

Pseudo-doubling can be differentiated from true doubling of the optic nerve by the imaging techniques of fluorescein angiography, ultrasonography, computerized tomography (CT), and magnetic resonance imaging (MRI). Such a true doubling of the optic disc - where there are two optic nerve heads and two associated optic discs in one eye - is extremely rare ${ }^{1,4-5}$.

In this case, the patient reports no symptoms other than strain at near when using the VDU screen. A near refractive prescription was given to her for prolonged near activities. She was advised on appropriate lid hygiene measures to reduce the blepharitis noted during slitlamp biomicroscopy. A referral letter was given to her for ophthalmological review, but she did not seem to be too interested in attending such an appointment and at the time of submission of this case review had not sought ophthalmological opinion.

\section{References}

1. Pesudovs K, Weisinger HS. Pseudoduplication of the optic nerve head. Optometry 200071 586-590.

2. McLoone EM, Buchanan TA. Duplication of the optic disc: true or pseudo? A coloboma or not a coloboma? Eur J Ophthalmol 200414 163-165.

3. Islam N, Best J, Mehta JS, Sivakumar S, Plant GT, Hoyt WF. Optic disc duplication or coloboma? Br J Ophthalmol 200589 26-29.

4. Kamath GG, Prasad SP, Patwala YJ, Watts MT. Peripapillary coloboma simulating double optic disc. Br J Ophthalmol 199983 1207-1209.

5. Taylor D, Stout A. Optic nerve: Congenital abnormalities In: Taylor D. (ed) Paediatric Ophthalmology, (2nd edition). Oxford: Blackwell Science Limited. 1997 pp. 682-684.

6. Barboni P, Deluigi M, De Bonis C, Monetti G, Savini G, Zanini M. Pseudodoubling of the optic disc. Arch Ophthalmol 1998116 1400-1401.

7. Brink JK, Larson FE. Pseudodoubling of the optic disc: a fluorescein angiorgraphic study of a case with coloboma. Acta Ophthalmol 197755 862-870. 